Article

\title{
Chemical Composition and Antimicrobial Activities of Artemisia argyi Lévl. et Vant Essential Oils Extracted by Simultaneous Distillation-Extraction, Subcritical Extraction and Hydrodistillation
}

\author{
Xiao Guan ${ }^{1, *}$, Depeng Ge ${ }^{1}$, Sen $\mathrm{Li}^{1}$, Kai Huang ${ }^{1}$, Jing Liu ${ }^{2}$ and Fan $\mathrm{Li}^{1}$ \\ 1 School of Medical Instruments and Food Engineering, University of Shanghai for Science and Technology, \\ Shanghai 200093, China; gedepeng@hotmail.com (D.G.); lisen_1027@126.com (S.L.); \\ hkjn1990@163.com (K.H.); hkshlg2018@163.com (F.L.) \\ 2 College of Information Engineering, Shanghai Maritime University, Shanghai 200135, China; \\ jingliu@shmtu.edu.cn \\ * Correspondence: gnxo@hotmail.com; Tel.: +86-021-5539-6693
}

Received: 27 December 2018; Accepted: 28 January 2019; Published: 29 January 2019

check for updates

\begin{abstract}
Artemisia argyi Lévl. et Vant essential oil could be used as a good antimicrobial flavouring agent and applied in the food industry. In this study, three methods, including simultaneous distillation-extraction (SDE), subcritical extraction and hydrodistillation, were applied to extract $A$. argyi essential oil. Compared with subcritical extraction (1\%) and hydrodistillation $(0.5 \%)$, SDE gave a higher yield $(1.2 \%)$. Components of the essential oils were analysed with gas chromatography-mass spectrometry (GC-MS), and the most abundant ingredients were caryophyllene oxide, neointermedeol, borneol, $\alpha$-thujone and $\beta$-caryophyllene. These five components accounted for $82.93 \%, 40.90 \%$ and $40.33 \%$ for SDE, subcritical extraction, and hydrodistillation, respectively. Based on agar disc diffusion and minimum inhibitory concentration (MIC) assays, SDE oil showed a significant inhibitory effect towards Listeria monocytogenes, Escherichia coli, Proteus vulgaris, Salmonella enteritidis and Aspergillus niger. Furthermore, electron microscope observations (SEM) confirmed that SDE oil could obviously deform cell morphology and destroy the structure of cell walls. Performances showed that SDE was a promising process for extracting $A$. argyi essential oil with both high yield and antimicrobial activity.
\end{abstract}

Keywords: Artemisia argyi; simultaneous distillation extraction; essential oil; antimicrobial activity

\section{Introduction}

Artemisia argyi, known as Chinese mugwort, is an herbaceous perennial plant with creeping rhizomes. A. argyi is the most popular plant in China and eastern Asia, and its leaves are used as a Traditional Chinese Medicine (TMC). Artemisia species are important medical plants which have aroused interest for their biological and chemical diversities [1]. Known for a long time for the treatment of diseases such as asthma, inflammation, hepatitis and infections by bacteria, fungi or viruses [2], A. capillaris herba has officially been listed in the Chinese Pharmacopoeia and applied in the treatment of epidemic hepatitis [3]. In addition, in Europe, the use of A. mexicana to stimulate the appetite and to aid digestion is allowed [4].

The main pharmacological active compound in A. argyi is the essential oil and the biological activities of the extracted essential oil have been investigated. For example, it has been reported that $A$. argyi essential oil showed anti-histaminic effects and antifungal activity [5]. Moreover, A. argyi volatile oil had strong antibacterial effects against Staphylococcus aureus, Escherichia coli and Salmonella enteritidis [6]. Gas chromatography-mass spectrometry (GC-MS) has been proved to be an 
useful method for the determination of the different essential oil components. Recently, the composition of essential oil extracted from leaves and flowers of $A$. argui has been reported [7], and the major components were sesquiterpenes, monoterpenes, alcohols, ketones, aromatic compounds esters and ethers, etc. Based on the appealing aromatic properties, A. argui essential oil could play a significant role in food safety and preservation [8]. At present, most preservatives used by the food industry are artificial additives such as nitrates, sulphur dioxide and benzoates [9]. Natural compounds may have great commercial value in the food industry, however their applications are currently limited owing to their high cost. Hence, an effective and low-cost preparation method is needed.

Classical methods of extraction, such as solid-liquid extraction with organic solvents, are used for the extraction of lipid fractions and essential oils from aromatic plants. These solvents provide good recovery of oil and other compounds, but they have certain drawbacks such as potential human and environmental toxicity. Compared with the classical methods, hydrodistillation has been the most common method used to extract the essential oils from plants. Although this method still has drawbacks such as long extraction times and high energy use, hydrodistillation is the simplest and most practical method [10,11]. Recently, there has been great interest in subcritical extraction for its mild operational temperature, no solvent residues and better nutrition retention. Because subcritical solvents have the advantages of high density, high diffusively and low viscosity, the subcritical extraction method has been widely applied in lipid and essential oil extraction [12]. Furthermore, it is reported that simultaneous distillation-extraction (SDE) could be also used in essential oil extraction. This one-step extraction technique is less time consuming and allows a greater reduction of solvent volumes due to the continuous recycling. Under certain conditions a higher yield and richer ingredients could be achieved and the extracts obtained by SDE are free from non-volatile materials such as cuticular waxes and chlorophylls $[13,14]$. Recently, our laboratory has focused on searching for valuable plant essential oils which could be applied in the food safety and preservation. Up to now, A. argyi essential oils extracted by simultaneous distillation have never been reported. In this study, we examined different methods, including SDE, subcritial extraction and hydro-distillation, for $A$. argyi essential oil extraction and characterized the corresponding compositions. The aim of this study was to evaluate the effects of these methods on the yield and the biological characteristics of the essential oil, and to analyze its inhibitory effects on microorganisms that cause vegetable, fruit and other food losses.

\section{Results and Discussion}

\subsection{Extraction Yields of A. argyi Essential Oils}

A. argyi essential oils were extracted with hydrodistillation, subcritical extraction and SDE respectively. Their extraction time, yields and colors were evaluated. As shown in Table 1, SDE took the shortest time (180 $\mathrm{min})$ and gave the highest yield (1.2\%), followed by subcritical extraction (1\%) and hydrodistillation $(0.5 \%)$. The yields of these extracted oils were higher than the reported ones, which were around $0.20 \%$ to $0.26 \%$ [15]. Furthermore, SDE cost $1 \mathrm{~h}$ less than hydrodistillation, and $2 \mathrm{~h}$ less than subcritical extraction. Compared with the other studies, SDE is a feasible method which offers yield and processing time advantages.

Table 1. Times and yields of Artemisia argyi essential oils obtained by three different extraction methods.

\begin{tabular}{cccc}
\hline Extraction Method & Extraction Time (min) & Yield (\% Dry Weight) & Colour \\
\hline Hydrodistillation & 240 & $0.50 \%$ & Dark green \\
Subcritical extraction & 300 & $1 \%$ & Yellow \\
SDE & 180 & $1.20 \%$ & Pale yellow \\
\hline
\end{tabular}

\subsection{Compositions of A. argyi Essential Oils}

The identified components of $A$. argyi essential oils obtained from the three different extraction methods and their concentrations are shown in Table 2. 
Table 2. Chemical compositions (in percent) of Artemisia argyi essential oils obtained by three different methods.

\begin{tabular}{|c|c|c|c|c|}
\hline \multirow[b]{2}{*}{ Compounds } & \multirow[b]{2}{*}{ CAS } & \multicolumn{3}{|c|}{ Relative Content (\%) } \\
\hline & & Hydrodistillation & $\begin{array}{l}\text { Subcritical } \\
\text { Extraction }\end{array}$ & SDE \\
\hline Monoterpene hydrocarbons & & 0.87 & 1.61 & 14.55 \\
\hline$\gamma$-Terpinene & $000099-85-4$ & 0.247 & 0.468 & - \\
\hline o-Cymene & $000527-84-4$ & 0.315 & 0.567 & - \\
\hline Terpinolene & $000586-62-9$ & 0.079 & 0.154 & - \\
\hline$\alpha$-Thujene & $002867-05-2$ & 0.233 & 0.42 & 14.551 \\
\hline Oxygenated monoterpenes & & 47.38 & 61.29 & 45.49 \\
\hline 2,5,5-Trimethyl-2,6-heptadien-4-one & $000512-37-8$ & 0.048 & 0.569 & - \\
\hline Yomogi alcohol & 026127-98-0 & 0.535 & 2.877 & - \\
\hline$\alpha$-Thujone & $000546-80-5$ & 7.989 & 11.312 & 14.551 \\
\hline$\beta$-Thujone & $000471-15-8$ & 1.916 & 1.928 & - \\
\hline trans-Sabinene hydrate & 017699-16-0 & 1.199 & 0.653 & - \\
\hline 2,2,4-Trimethyl-3-cyclohexene-1-carbaldehyde & $001726-47-2$ & - & 0.15 & - \\
\hline$(+)-2$-Bornanone & $000464-49-3$ & 3.896 & 7.253 & 10.022 \\
\hline trans-Pinocamphone & $000547-60-4$ & 0.179 & 0.216 & - \\
\hline Umbellulone & $024545-81-1$ & 0.407 & 0.123 & - \\
\hline cis-2-Menthenol & 029803-82-5 & 1.614 & - & - \\
\hline trans-Chrysanthenyl acetate & 054324-99-1 & - & 0.156 & - \\
\hline Bornyl acetate & $000076-49-3$ & 0.24 & 0.296 & - \\
\hline Dill ether & $070786-44-6$ & 0.059 & 0.087 & - \\
\hline 1-Terpinen-4-ol & $000562-74-3$ & 4.441 & 0.233 & - \\
\hline trans-Dihydrocarvone & 005948-04-9 & 0.252 & - & - \\
\hline Benihinal & 000564-94-3 & 0.275 & 0.464 & - \\
\hline trans-2,8-p-Mentha-dien-1-ol & $007212-40-0$ & 0.102 & 0.173 & - \\
\hline cis-2-Menthenol & 029803-82-5 & - & 2.351 & - \\
\hline (-)-trans-Pinocarveol & $000547-61-5$ & 0.349 & 0.835 & - \\
\hline Verbenol & 000473-67-6 & 0.209 & 0.153 & 1.827 \\
\hline Borneol & 000507-70-0 & 6.482 & 8.273 & 16.356 \\
\hline cis-Sabinol & 003310-02-9 & 5.505 & 1.747 & - \\
\hline Verbenone & $000080-57-9$ & - & 0.913 & - \\
\hline Isothujol & 000513-23-5 & 1.193 & - & - \\
\hline$\alpha$-Terpineol & 000098-55-5 & 3.617 & 4.119 & - \\
\hline Piperitone & 000089-81-6 & 0.422 & 0.49 & - \\
\hline$\alpha$-Phellandren-8-ol & $001686-20-0$ & - & 0.932 & - \\
\hline cis-Chrysanthenol & 055722-60-6 & 2.529 & 4.209 & 2.738 \\
\hline trans-Piperitol & $016721-39-4$ & 0.602 & 1.062 & - \\
\hline Myrtenol & $000515-00-4$ & - & 0.369 & - \\
\hline trans- $p$-Mentha-1(7),8-dien-2-ol & 021391-84-4 & 0.436 & 0.911 & - \\
\hline 4-Isopropyl-1,5-cyclohexadiene-1-methanol & $019876-45-0$ & 0.123 & 0.063 & - \\
\hline Dihydrocarveol & 000619-01-2 & 0.614 & 0.468 & - \\
\hline cis-Carveol & 001197-06-4 & 1.404 & 2.997 & - \\
\hline p-Cymene-8-ol & 001197-01-9 & 0.425 & 0.707 & - \\
\hline trans-Shisool & $022451-48-5$ & 0.01 & - & - \\
\hline$\beta$-Ionone & 000079-77-6 & 0.028 & 4.13 & - \\
\hline p-Isopropylbenzyl alcohol & $000536-60-7$ & 0.17 & 0.072 & - \\
\hline Thymol & $000089-83-8$ & 0.108 & - & - \\
\hline Sesquiterpene hydrocarbons & & 12.702 & 26.846 & 13.687 \\
\hline$\alpha$-Cubebene & 017699-14-8 & 0.079 & 0.124 & - \\
\hline (-)-Cyperene & $002387-78-2$ & 0.063 & 0.094 & - \\
\hline$\beta$-Bourbonene & $005208-59-3$ & 0.146 & 0.319 & - \\
\hline$\beta$-Ylangene & 020479-06-5 & 0.157 & 0.196 & - \\
\hline$\beta$-Caryophyllene & $000087-44-5$ & 7.495 & 20.022 & 13.687 \\
\hline$\alpha$-Humulene & 006753-98-6 & 2.236 & 2.339 & - \\
\hline$a$-Cyperene & $002387-78-2$ & 0.283 & - & - \\
\hline Alloaromadendrene & $025246-27-9$ & 0.139 & 0.18 & - \\
\hline Germacrene D & 037839-63-7 & 0.548 & 1.535 & - \\
\hline$\beta$-Selinene & $017066-67-0$ & 1.112 & 1.713 & - \\
\hline Longifolene & 000475-20-7 & 0.359 & - & - \\
\hline$\delta$-Cadinene & 000483-76-1 & - & 0.324 & - \\
\hline trans-Calamenene & $073209-42-4$ & 0.036 & - & - \\
\hline Chamazulene & 000529-05-5 & 0.049 & - & - \\
\hline Oxygenated sesquiterpenes & & 22.72 & 2.66 & 40.82 \\
\hline Caryophyllene oxide & 001139-30-6 & 8.713 & 0.133 & 21.553 \\
\hline Salvial-4(14)-en-1-one & 073809-82-2 & - & 0.133 & - \\
\hline$\alpha$-Humulene epoxide II & 019888-34-7 & 0.763 & 0.3 & - \\
\hline Junenol & 000472-07-1 & 0.22 & 0.128 & - \\
\hline Nerolidol & 000142-50-7 & 0.055 & - & - \\
\hline Spathulenol & $006750-60-3$ & 1.508 & 0.221 & 2.487 \\
\hline Neointermedeol & $005945-72-2$ & 9.652 & 1.16 & 16.779 \\
\hline
\end{tabular}


Table 2. Cont

\begin{tabular}{|c|c|c|c|c|}
\hline \multirow[b]{2}{*}{ Compounds } & \multirow[b]{2}{*}{ CAS } & \multicolumn{3}{|c|}{ Relative Content (\%) } \\
\hline & & Hydrodistillation & $\begin{array}{l}\text { Subcritical } \\
\text { Extraction }\end{array}$ & SDE \\
\hline $\begin{array}{l}\text { 11,11-Dimethyl-4,8-dimethylene- } \\
\text { bicyclo[7.2.0]undecan-3-ol }\end{array}$ & 079580-01-1 & 0.304 & 0.06 & - \\
\hline $\begin{array}{l}\text { 10,10-Dimethyl-2,6-dimethylene- } \\
\text { bicyclo[7.2.0]undecan-5.beta.-ol }\end{array}$ & 019431-80-2 & 1.302 & 0.443 & - \\
\hline Costol & $000515-20-8$ & 0.06 & 0.08 & - \\
\hline Phytol (3,7,11,15-Tetramethyl-2-hexadecen-1-ol) & 000150-86-7 & 0.14 & - & - \\
\hline Others & & 2.88 & 2.51 & 0 \\
\hline cis-Sabinyl acetate & $139757-62-3$ & 0.508 & 0.41 & - \\
\hline iso-Thujol & $007712-79-0$ & 0.734 & 0.545 & - \\
\hline Bornyl isovalerate & 000076-50-6 & 0.652 & 0.596 & - \\
\hline Bornyl tiglate & $000076-49-3$ & 0.404 & 0.38 & - \\
\hline 2-Methoxy-3-(2-propen-1-yl)phenol, & $001941-12-4$ & 0.209 & 0.433 & - \\
\hline Palmitic acid & $000057-10-3$ & 0.373 & 0.148 & - \\
\hline
\end{tabular}

The contents of compounds varied markedly along with the extraction method. As shown in Table 2, a total of 65, 62 and nine compounds were identified for the essential oil extracted with hydrodistillation, subcritical extraction and SDE, accounting for the $86.318 \%, 94.496 \%$ and $99.997 \%$ of total oil, respectively. Different from SDE oil, subcritical extraction oil and hydrodistillation oil were dominated by the monoterpene fractions with $62.479 \%$ and $48.021 \%$, respectively. However, the total content of monoterpene fractions in SDE oil was $45.49 \%$ and no monoterpene hydrocarbon fractions were determined. On the other hand, SDE oil was dominated by the sesquiterpene fractions, and large amount of the oxygenated sesquiterpene (40.82\%) was found. Although different extraction methods produced essential oils with various chemical compositions, there were five major compounds in common, including caryophyllene oxide, neointermedeol, borneol, $\alpha$-thujone and $\beta$-caryophyllene. As shown in Figure 1, the total of these five compounds accounted for around $82.93 \%$, $40.90 \%$ and $40.33 \%$ for SDE, subcritical extraction and hydrodistillation, respectively. Similarly, it is well-documented that caryophyllene oxide, neointermedeol, borneol, $\alpha$-thujone and $\beta$-caryophyllene had the highest contents [16]. Mevy et al. have proved that these components exhibited antioxidant, antibacterial and other biological activities [17]. Additionally, compared with the other two methods, the contents of these common ingredients in SDE essential oil was obviously higher. Usually, traditional essential oil extractions are time consuming and high temperature needed. These methods might lose some volatiles and significantly degrade unsaturated compounds or esters [18]. Similarly, SDE would also result in severe losses of volatile materials, because oil-containing hexane should be subsequently removed by rotary evaporation.

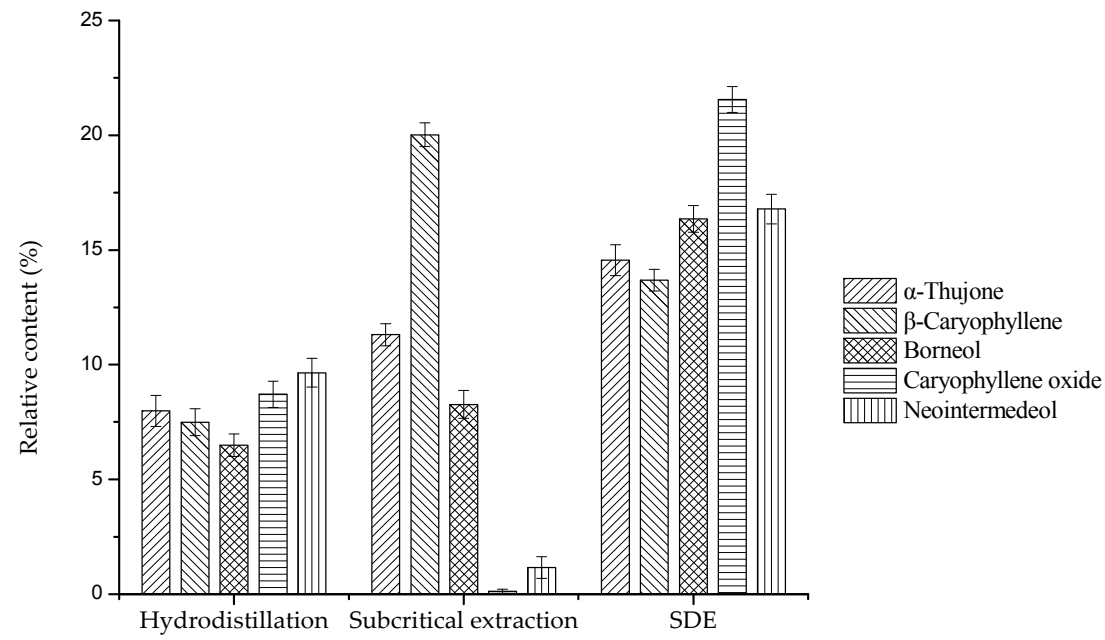

Figure 1. The major chemical components of Artemisia argyi essential oils (in percentages, average of a duplicate assay). 


\subsection{Antimicrobial Activities of A. argyi Essential Oils}

The antimicrobial activities of $A$. argyi essential oils determined by the agar disc diffusion assay are listed in Table 3. In general, Gram-positive pathogens were much more sensitive to A. argyi essential oil than fungi and Gram-negative pathogens. Bacillus subtilis was the most sensitive bacterium, with an inhibition diameter longer than $15 \mathrm{~mm}$ and Escherichia coli was the most inhibited Gram-negative bacterium with an inhibition diameter longer than $9.7 \mathrm{~mm}$. As shown in Figure 2, hydrodistillation oil showed strong inhibition against $S$. aureus and B. subtilis, whereas subcritical extraction oil showed significant inhibition against B. subtilis and S. cerevisiae, with inhibition zone diameters of 21.88 and $14.7 \mathrm{~mm}$, respectively.

Table 3. Inhibition zones (diameter, $\mathrm{mm}$ ) of Artemisia argyi essential oils against various microorganisms.

\begin{tabular}{cccc}
\hline \multirow{2}{*}{ Microorganisms } & \multicolumn{3}{c}{ Extraction Method } \\
\cline { 2 - 4 } & Hydrodistillation & Subcritical Extraction & SDE \\
\hline Staphylococcus aureus & $18.23^{\mathrm{a}} \pm 0.26$ & $8^{\mathrm{g}} \pm 0.19$ & $13.23^{\mathrm{cd}} \pm 0.37$ \\
Bacillus subtilis & $17.43^{\mathrm{b}} \pm 0.45$ & $21.88^{\mathrm{a}} \pm 0.39$ & $15.15^{\mathrm{b}} \pm 0.44$ \\
Listeria monocytogenes & $13.28^{\mathrm{c}} \pm 0.51$ & $8.3^{\mathrm{g}} \pm 0.58$ & $13.43^{\mathrm{c}} \pm 0.61$ \\
Escherichia coli & $10.03^{\mathrm{d}} \pm 0.38$ & $9.7^{\mathrm{e}} \pm 0.41$ & $14.78^{\mathrm{b}} \pm 0.46$ \\
Proteus vulgaris & $9.35^{\mathrm{e}} \pm 0.66$ & $10.63^{\mathrm{d}} \pm 0.58$ & $15.8^{\mathrm{a}} \pm 0.46$ \\
Salmonella enteritidis & $10.48^{\mathrm{d}} \pm 0.54$ & $8.93^{\mathrm{f}} \pm 0.66$ & $13.95^{\mathrm{c}} \pm 0.39$ \\
Saccharomyces cerevisiae & $13.53^{\mathrm{c}} \pm 0.58$ & $14.7^{\mathrm{b}} \pm 0.52$ & $11.23^{\mathrm{f}} \pm 0.49$ \\
Aspergillus niger & $10.45^{\mathrm{d}} \pm 0.61$ & $11.3^{\mathrm{c}} \pm 0.53$ & $12.53^{\mathrm{e}} \pm 0.46$ \\
\hline
\end{tabular}

Data are average of a duplicate test (mean \pm SD) with at least three determinations. ${ }^{a, b, c, d, e, f, g}$ : Values with different superscripts are significantly different in line $(p<0.5)$. Negative control inhibition diameter: not detected.

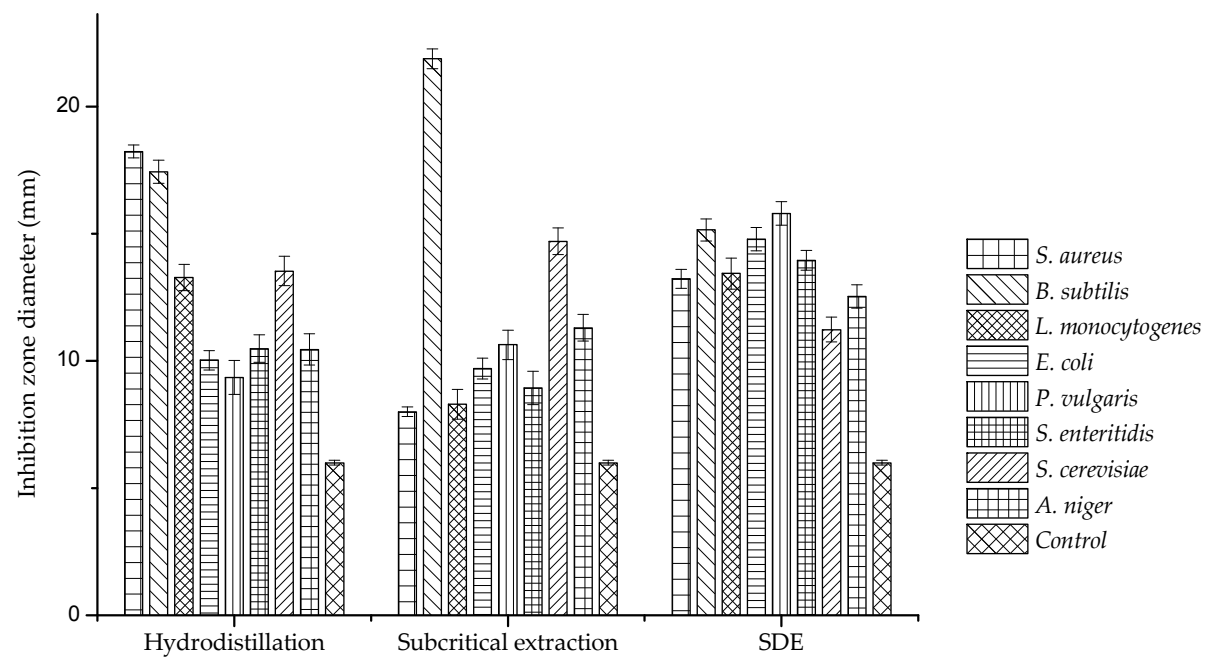

Figure 2. Inhibition zones of Artemisia argyi essential oils against various microorganisms.

Interestingly, SDE oil showed a broad spectrum of antimicrobial activity with a $13 \mathrm{~mm}$ diameter on average. Furthermore, MICs of different $A$. argyi essential oils against eight microbes were also tested (listed in Table 4 and shown in Figure 3). MICs of SDE oil against most microorganisms were $6.25 \mu \mathrm{L} / \mathrm{mL}$, peaking at $12.5 \mu \mathrm{L} / \mathrm{mL}$. In contrast, MICs of hydrodistilled and subcritical extracts were mostly higher than $12.5 \mu \mathrm{L} / \mathrm{mL}$ and several values even reached $25 \mu \mathrm{L} / \mathrm{mL}$. As the results above indicate, SDE oil showed a higher antimicrobial activity than the other two oils, which suggested that SDE oil could be a good candidate for an antimicrobial agent. Danh et al. reported that the antimicrobial activity of $A$. argyi essential oil was predominantly controlled by the amount of active compound with high diffusivity in agar medium [19]. During hydrodistillation and subcritical extraction, compounds could be hardly diffused, and those with high hydrophobicities exerted little influence on the antimicrobial activities of resultant oils [20]. The antimicrobial activities of 
essential oils were apparently related to the large amount of caryophyllene oxide, neointermedeol, borneol, $\alpha$-thujone and $\beta$-caryophyllene. The activity of SDE oil was superior to those of subcritical and hydrodistilled extracts, which displayed lower MICs and larger inhibition zones. Compared with SDE, hydrodistillation needs large quantity of water and higher temperature, which would cause hydrolysis reaction and damage the active compound. Subcritical extraction would bring some miscellaneous ingredients such as wax and pigment. These ingredients might be the reason for the less inhibitory effect of essential oil extracted by subcritical extraction [12]. On the other hand, the highest antimicrobial activity of essential oil extracted by SDE might also be related with $n$-hexane which has the appropriate polarity to accumulate active antimicrobial ingredients.

Table 4. MICs $(\mu \mathrm{L} / \mathrm{mL})$ of Artemisia argyi essential oils against various microorganisms.

\begin{tabular}{cccc}
\hline \multirow{2}{*}{ Microorganisms } & \multicolumn{3}{c}{ Extraction Method } \\
\cline { 2 - 4 } & Hydrodistillation & Subcritical Extraction & SDE \\
\hline Staphylococcus aureus & $6.25^{\mathrm{c}} \pm 0.38$ & $12.5^{\mathrm{b}} \pm 0.37$ & $12.5^{\mathrm{a}} \pm 0.37$ \\
Bacillus subtilis & $6.25^{\mathrm{c}} \pm 0.52$ & $3.13^{\mathrm{d}} \pm 0.45$ & $12.5^{\mathrm{a}} \pm 0.42$ \\
Listeria monocytogenes & $6.25^{\mathrm{c}} \pm 0.37$ & $25^{\mathrm{a}} \pm 0.41$ & $6.25^{\mathrm{b}} \pm 0.53$ \\
Escherichia coli & $12.5^{\mathrm{b}} \pm 0.39$ & $12.5^{\mathrm{b}} \pm 0.48$ & $6.25^{\mathrm{b}} \pm 0.51$ \\
Proteus vulgaris & $25^{\mathrm{a}} \pm 0.43$ & $12.5^{\mathrm{b}} \pm 0.37$ & $6.25^{\mathrm{b}} \pm 0.41$ \\
Salmonella enteritidis & $12.5^{\mathrm{b}} \pm 0.42$ & $25^{\mathrm{a}} \pm 0.34$ & $6.25^{\mathrm{b}} \pm 0.52$ \\
Saccharomyces cerevisiae & $6.25^{\mathrm{c}} \pm 0.45$ & $6.25^{\mathrm{c}} \pm 0.4$ & $12.5^{\mathrm{a}} \pm 0.37$ \\
Aspergillus niger & $12.5^{\mathrm{b}} \pm 0.66$ & $12.5^{\mathrm{b}} \pm 0.51$ & $6.25^{\mathrm{b}} \pm 0.36$ \\
\hline
\end{tabular}

Data are average of a duplicate (mean $\pm \mathrm{SD}$ ). ${ }^{\mathrm{a}, \mathrm{b}, \mathrm{c}}$ : Values with different superscripts are significantly different in line $(p<0.5)$.

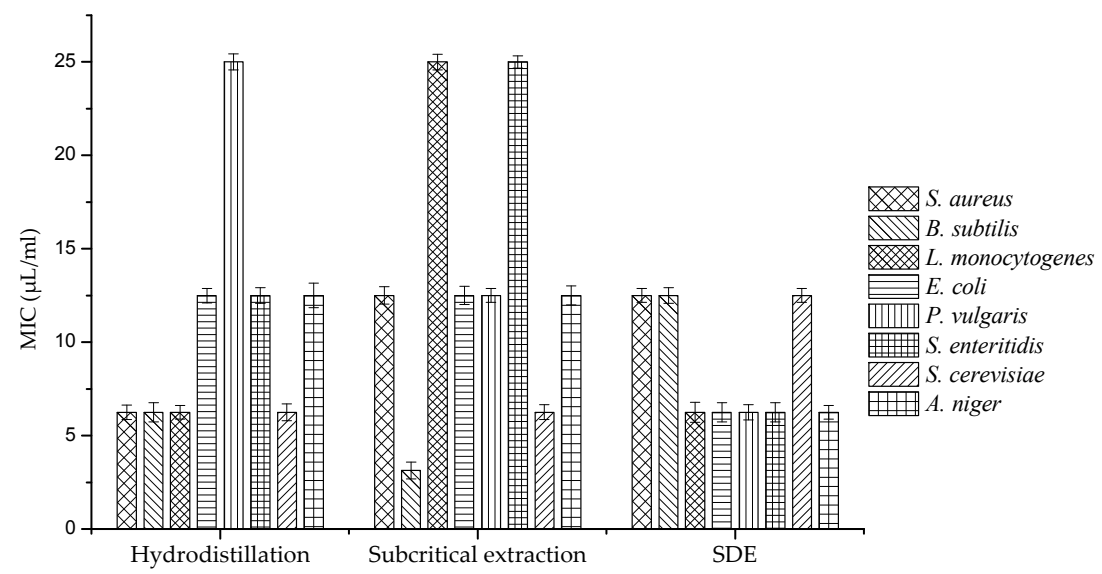

Figure 3. MICs of Artemisia argyi essential oils against various microorganisms.

Taking both of the results from MICs and inhibition zone diameter into consideration, S. aureus and E. coli were the appropriate target bacteria with higher sensitivity. To further investigate the antimicrobial activity of the extracted essential oils, SEM analysis was performed using the two selected sensitive bacteria. As shown in Figures 4 and 5, the images directly exhibited the detrimental inhibitory effect of the essential oil from $A$. argyi against the tested bacteria. It could be found that, for the most part, control cells were intact and showed a smooth surface or weakly damaged (Figure 4(A4) and Figure 5(B4)). In contrast, bacterial cells treated with the A. argyi essential oils were subjected to considerable damage (Figure 4(A1-A3) and Figure 5(B1-B3)). Similar results have been reported by Diao et al. [21]. These results revealed that the active compounds from essential oil might bind to the cell surface and then penetrate into the target sites, which could destroy the structure of cell walls. It should be noticed that, as shown in Figures 4 and 5, the cell walls changed much to different extents after treated with the essential oil, while SDE oil showed the strongest inhibitory effect against the strains. 

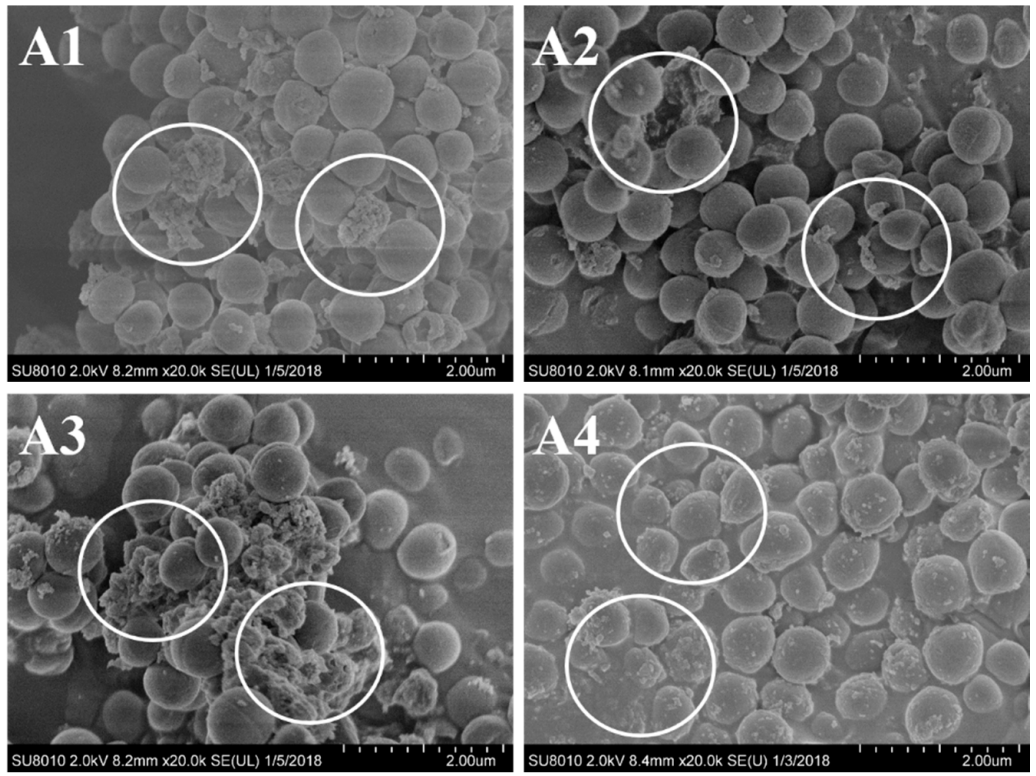

Figure 4. SEM images of $S$. aureus treated with Artemisia argyi essential oils obtained by hydrodistillation (A1), subcritical extraction (A2) and SDE (A3) at the MICs, respectively. And (A4) is control sample.
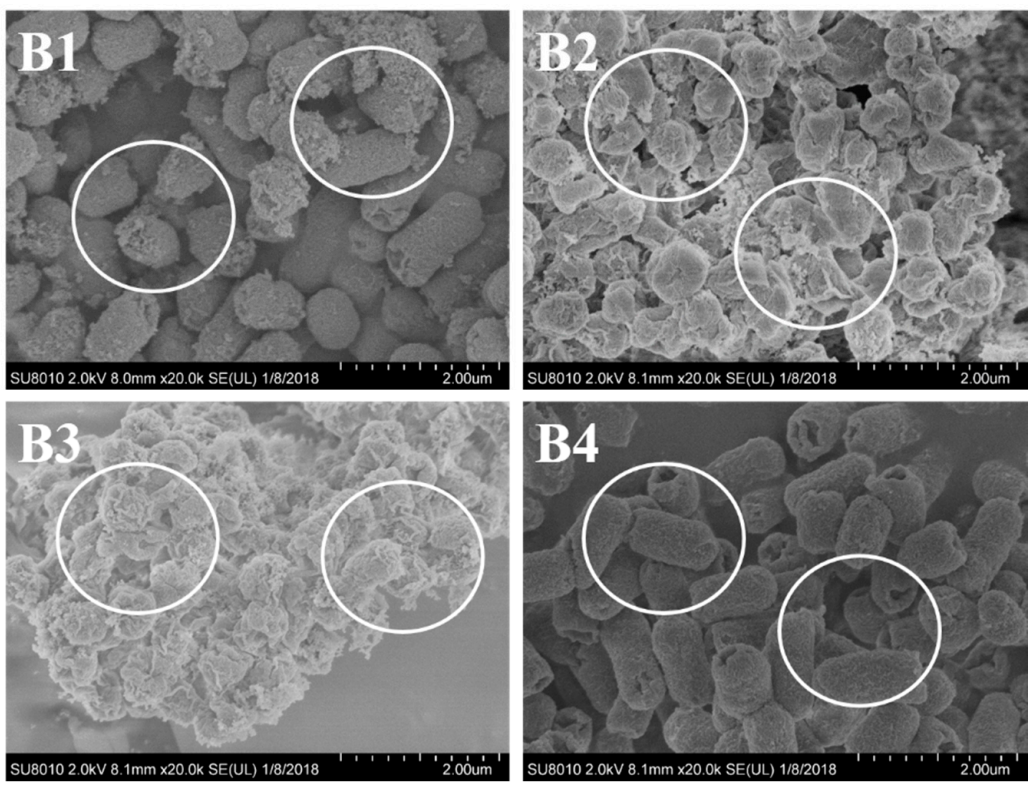

Figure 5. SEM images of E. coli treated with Artemisia argyi essential oils obtained by hydrodistillation (B1), subcritical extraction (B2) and SDE (B3) at the MICs, respectively. And (B4) is control sample.

\section{Materials and Methods}

\subsection{Materials}

Leaves of $A$. argyi Lévl. et Vant were purchased from an A. argyi farm (Qichun, China). The plant was identified by ProfessorXu Fei, from the School of Food Science and Technology, University of Shanghai for Science and Technology. The leaves were ground into powders and stored at $4{ }^{\circ} \mathrm{C}$.

\subsection{Strains}

The antimicrobial activities of essential oil samples were tested against eight different microorganisms including three Gram-positive bacteria Staphylococcus aureus (ATCC 6538), Bacillus subtilis (CMCC B63501) 
and Listeria monocytogenes (ATCC 19115), three Gram-negative bacteria Escherichia coli (ATCC 12900), Proteusbacillus vulgaris (CMCC B49027) and Salmonella enteritidis (ATCC 13076), as well as two fungi: Saccharomyces cerevisiae (ATCC 9763) and Aspergillus niger (ATCC 6275). These strains were obtained from the School of Medical Instrument and Food Engineering, University of Shanghai for Science and Technology (Shanghai, China), and stored in glass ampoules at $-80{ }^{\circ} \mathrm{C}$ prior to use. In this test, nutrient broth (NB), potato dextrose agar (PDA), lauryl sulfate tryptose broth (LSTB), Czapek dox agar (CDA), yeast extract dextrose chloramphenicol agar (YEDCA) and modified Czapek dox broth (MCDB) medium were used as culture media, and there were purchased from Sinopharm Chemical Reagent Co., Ltd. (Shanghai, China).

\subsection{Extraction of Essential Oils}

\subsubsection{Hydrodistillation}

Ground A. argyi samples (100 g) mixed with $1500 \mathrm{~mL}$ deionized water were hydrodistilled in a glass Clevenger-type apparatus for $4 \mathrm{~h}$. Details of the procedures were followed as the previous report [22]. Extracted oil was weighed and stored at $4{ }^{\circ} \mathrm{C}$, and the experiment was repeated three times.

\subsubsection{Subcritical Extraction}

The subcritical extraction system (shown in Figure 6) was provided by Henan Subcritical Biological Technology Co., Ltd. (Henan, China). In this study, $100 \mathrm{~g}$ ground A. argyi sample was loaded into the extraction cell. Butane from the storage pot (No. 1) was passed to refrigerator to be cooled and liquefied. Then liquefied butane was pressurized by the compressor (No. 7). Subsequently, compressed butane was passed into the solvent bottle (No. 4) and then transferred to the extraction agent (No. 2). In the shell-and-tube heat exchanger, water was circulated in its shell with constant temperature, providing the required temperature for extraction. The operation time was determined for all experiments by closing the extraction agent (No. 2) valve for about 5 h. The obtained essential oil was collected carefully from knockout drum (No. 3). Because the essential oil was sensitive to light and heat, it was carefully weighed and kept in a sealed murky vial in an ice box before analysis. According to the reported method [23], the experiment was repeated three times.
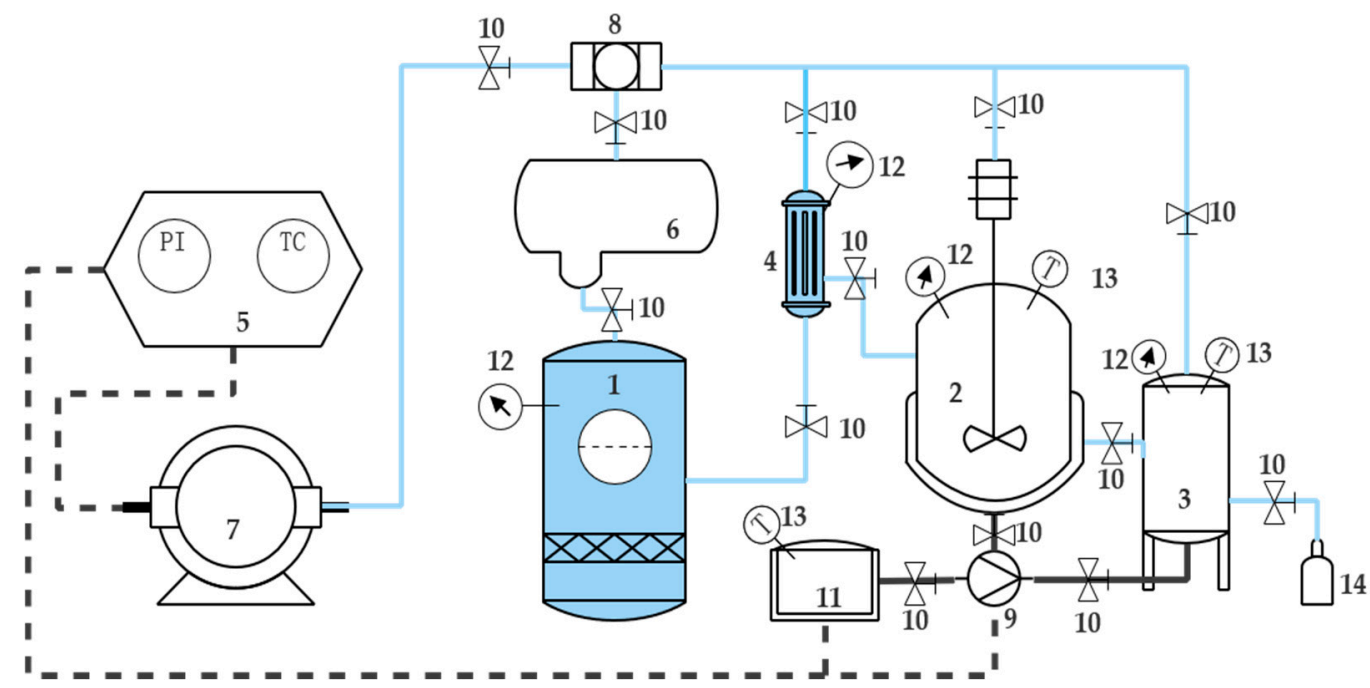

Figure 6. Schematic diagram of subcritical extraction experimental apparatus. Parts of the apparatus are numbered as follows: 1 . Butane solvent storage pot; 2 . Extraction agent; 3. Knockout drum; 4. Solvent bottle; 5 . Control panel; 6. Condenser; 7. Compressor; 8. Filter; 9. Water pump; 10. Spherical value; 11. Hot water tank; 12. Piezometer; 13. Thermometer; 14 . Collection bottle. 


\subsubsection{Simultaneous Distillation-Extraction (SDE)}

Simultaneous distillation-extraction of $A$. argyi essential oil was performed with a specially designed equipment (shown in Figure 7). Ground A. argyi sample (100 g) was loaded into the flask (extraction agent, No. 2) with $1500 \mathrm{~mL}$ deionized water, and coupled with another flask (No. 9) which contained $30 \mathrm{~mL}$ hexane. The operating temperatures of thermostats No. 1 and 10 were set at $100{ }^{\circ} \mathrm{C}$ and $45^{\circ} \mathrm{C}$ respectively. The extraction system was performed for $3 \mathrm{~h}$. Afterwards, the valve (No. 8) was turned on, and the continuously condensed distillate was collected in the receiver (No. 11). The essential oil and hexane were separated by a rotary evaporator. Subsequently, the extract was weighed and stored at $4{ }^{\circ} \mathrm{C}$. On the basis of the reported method [24], the experiment was repeated three times.

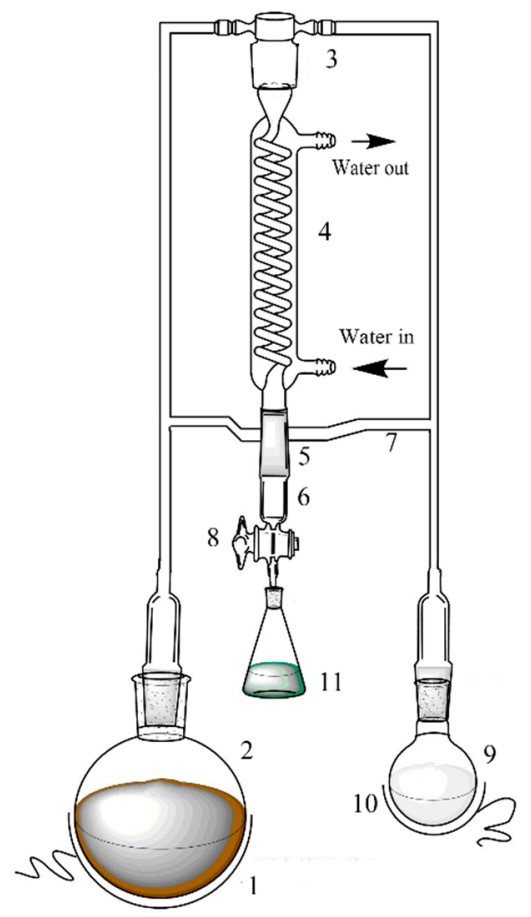

Figure 7. Schematic diagram of SDE experimental apparatus. Parts of the apparatus are numbered as follows: 1. Thermostat; 2. extraction agent (water); 3. mixing zone of water steam and n-hexane steam; 4. condenser; 5 . extraction cell; 6 . cooling jacket; 7 . phase separation tube; 8 . valve; 9 . reaction flask containing n-hexane; 10 . thermostat; 11 . collection bottle.

\subsubsection{Extraction Yield Calculation}

The extraction yields of $A$. argyi essential oil were calculated as follows:

$$
\text { Extraction yield }=\frac{\text { Weight of essential oil }}{\text { Weight of ground samples }} \times 100
$$

\subsection{Chemical Analysis of Essential Oil}

The GC-MS system (Agilent, Little Falls, DE, USA) was composed on a 5975 mass selective detector and a 6890 GC. The GC capillary column was an Agilent HP-5MS $(30 \mathrm{~m} \times 0.25 \mathrm{~mm} \times 0.25 \mu \mathrm{m})$. The injector and detector temperatures were 220 and $260{ }^{\circ} \mathrm{C}$, respectively. The oven temperature was held at $45^{\circ} \mathrm{C}$ for $5 \mathrm{~min}$, increased to $250^{\circ} \mathrm{C}$ with a flow rate of $3{ }^{\circ} \mathrm{C} / \mathrm{min}$, and then held for $10 \mathrm{~min}$. A $1 \mu \mathrm{L}$ aliquot of sample was injected in the split mode with a ratio of 1:10. Helium was used as the carrier gas with a flow rate of $1 \mathrm{~mL} / \mathrm{min}$.

Samples were analyzed by GC-MS and the data were analyzed using the MetAlign ${ }^{\mathrm{TM}}$. Software (version 3.0, Wageningen University, 2011, http:/ / www.MetAlign.nl). In this untargeted approach, 
the software was used to align and compare full scan GC-MS chromatograms and assess possible difference between samples. Raw data from GC-MS chromatograms were imported into MetAlign ${ }^{\mathrm{TM}}$, and baseline correction, denoising and smoothing of the data were carried out. This was achieved by setting parameters in the MetAlign ${ }^{\mathrm{TM}}$ interface, where the minimum peak threshold to eliminate noise that would interfere with peak alignment, was determined. Spectral alignment was performed using the rough mode, where ion fragments originating from chromatographic peaks with corresponding retention times were aligned across all samples.

\subsection{Detection of Antimicrobial Activity by Agar Disc Diffusion Assay}

The agar disc diffusion assay was carried out to evaluate the antimicrobial activities of the essential oils with different extraction methods. Briefly, $100 \mu \mathrm{L}$ aliquot of microbial inocula in the exponential growth phase (cell concentration of $10^{5}-10^{6} \mathrm{CFU} / \mathrm{mL}$ ) were spread over the plate surfaces. The three essential oils were dissolved in $1 \%$ dimethyl sulfoxide to $25 \mu \mathrm{L} / \mathrm{mL}$. Four sterile test discs $(\phi=6 \mathrm{~mm})$ were placed onto each agar plate and then injected with $10 \mu \mathrm{L}$ essential oil individually. Negative control was prepared using $1 \%$ dimethyl sulfoxide and the antibiotic amoxicillin was used as positive control at the concentration $50 \mu \mathrm{g} / \mathrm{mL}$. The plates were incubated for $24 \mathrm{~h}$ at $37^{\circ} \mathrm{C}$ for bacteria and for $48 \mathrm{~h}$ at $30^{\circ} \mathrm{C}$ for yeasts. Full diameter of the zone was regarded as the inhibition zone diameter, which was read by eye and measured by ruler. The tests were performed in four replicates for each sample.

\subsection{Measurement of Minimum Inhibitory Concentration (MIC)}

The three essential oils were first dissolved in $1 \%$ dimethyl sulfoxide and diluted to $50.000,25.000$, $12.500,6.250,3.125$, and $1.562 \mu \mathrm{L} / \mathrm{mL}$ respectively. Then $1 \mathrm{~mL}$ of each diluted sample was mixed with $14 \mathrm{~mL}$ of PDA culture medium and poured into a sterile petri dish. Subsequently, $1 \mathrm{~mL}$ culture medium was inoculated onto the potato dextrose agar plates and incubated for $48 \mathrm{~h}$ at $28^{\circ} \mathrm{C}$. The growth of the strains was monitored and the assay was performed in triplicate. The MIC was defined as the lowest concentration of the essential oil at which the microorganism tested did not demonstrated visible on the plates [25].

\subsection{Scanning Electron Microscope Observations}

SEM studies were carried out to observe the morphological changes of bacteria, which were treated with MIC value of $A$. argyi essential oil. Cells were harvested by centrifugation for $15 \mathrm{~min}$ at $5000 \mathrm{r} / \mathrm{min}$, and washed three times with $0.1 \mathrm{M}$ phosphate buffer solution (PBS, PH 7.4). The treatment of bacterial cells and observations of SEM was followed by the reported study [26]. All the samples were coated with gold in a sputter coater, followed by microscopic examinations using a SEM system (S-3000H; Hitachi Ltd., Tokyo, Japan).

\subsection{Statistical Analysis}

ANOVA was performed using Statistical Package for Social Sciences (SPASS 20.0, SPASS Inc., Carlsbad, CA, USA) for comparing mean values at $p<0.5$.

\section{Conclusions}

A. argyi essential oil has aroused attention due to its biological activities. In this study, three methods were applied to extract the essential oil from $A$. argyi leaves. The effects of each method on the yields, chemical composition and bioactivity were investigated. The yield of SDE extract was nearly $1.2 \%$, which was comparable to the subcritical extract (1\%), while much higher than the hydrodstilled oil (0.5\%). Although the chemical compounds of the SDE oil was much less than hydrodistilled oil and subcritical extract oil, the major compounds including caryophyllene oxide, neointermedeol, borneol, $\alpha$-thujone and $\beta$-caryophyllene of SDE oil accounted for $82.93 \%$, which was higher than subcritical extraction and hydrodistillation. The antimicrobial activities of these oils 
were estimated using agar disc diffusion and MIC assays. They showed that SDE oil had a good antimicrobial activity, and the activity against tested microorganisms like L. monocytogenes, E. coli, P. vulgaris, S. enteritidis and A. niger was higher or equal to those of other two extracts. Furthermore, SEM results showed that $A$. argyi essential oil could destroy the structure of cell walls and the SDE oil had the strongest inhibitory effect against the tested strains. All these results above suggested that SDE A. argyi essential oil had the advantages in yield, bioactive ingredients and antimicrobial activities, which qualified that SDE oil could be a good preservative candidate for the food industry.

Author Contributions: X.G. and D.G. conceived and designed the experiments; D.G. performed the experiments; D.G., K.H. and S.L. analyzed the data; J.L. contributed reagents/materials/analysis tools; X.G. and D.G. wrote the paper. F.L. revised the paper.

Funding: This work was supported by the National Natural Science Foundation of China (31701515), and Yangze River Delta Cooperation Program (18395810200).

Conflicts of Interest: No potential conflict of interest was reported by the authors.

\section{References}

1. Li, N.; Yu, M.; Zhang, X. Separation and Identification of Volatile Constituents in Artemisia argyi Flowers by GC-MS with SPME and Steam Distillation. J. Chromatogr. Sci. 2008, 46, 401-405. [CrossRef]

2. Mannan, A.; Ahmed, I.; Arshad, W.; Asim, M.F.; Qureshi, R.A.; Hussain, I.; Mirza, B. Survey of artemisinin production by diverse Artemisia species in northern Pakistan. Malar. J. 2010, 9, 310. [CrossRef] [PubMed]

3. Guo, F.Q.; Liang, Y.Z.; Xu, C.J.; Li, X.N.; Huang, L.F. Analyzing of the volatile chemical constituents in Artemisia capillaris herba by GC-MS and correlative chemometric resolution methods. J. Pharm. Biomed. Anal. 2004, 35, 469-478. [CrossRef] [PubMed]

4. Setzer, W.N.; Vogler, B.; Schmidt, J.M.; Leahy, J.G.; Rives, R. Antimicrobial activity of Artemisia douglasiana leaf essential oil. Fitoterapia 2004, 75, 192-200. [CrossRef] [PubMed]

5. Huang, H.-C.; Wang, H.-F.; Yih, K.-H.; Chang, L.-Z.; Chang, T.-M. Dual Bioactivities of Essential Oil Extracted from the Leaves of Artemisia argyi as an Antimelanogenic versus Antioxidant Agent and Chemical Composition Analysis by GC/MS. Int. J. Mol. Sci. 2012, 13, 14679. [CrossRef] [PubMed]

6. Smith-Palmer, A.; Stewart, J.; Fyfe, L. The potential application of plant essential oils as natural food preservatives in soft cheese. Food Microbiol. 2001, 18, 463-470. [CrossRef]

7. Yang, H.; Yang, Y.; Yin, N.; Wang, C.; Tong, Z. Facile preparation of artemisia argyi oil-loaded antibacterial microcapsules by hydroxyapatite-stabilized Pickering emulsion templating. Colloids Surf. B Biointerfaces 2013, 112, 96-102. [CrossRef]

8. Wang, W.; Zhang, X.K.; Wu, N.; Fu, Y.-J.; Zu, Y.-G. Antimicrobial activities of essential oil from Artemisiae argyi leaves. J. For. Res. 2006, 17, 332-334. [CrossRef]

9. Saguy, I.; Mannheim, C.H.; Passy, N. The role of sulphur dioxide and nitrate on detinning of canned grapefruit juice. Int. J. Food Sci. Technol. 2010, 8, 147-155. [CrossRef]

10. Cui, Q.; Wang, L.T.; Liu, J.Z.; Wang, H.M.; Guo, N.; Gu, C.B.; Fu, Y.J. Rapid extraction of Amomum tsao-ko essential oil and determination of its chemical composition, antioxidant and antimicrobial activities. J. Chromatogr. B Anal. Technol. Biomed. Life Sci. 2017, 1061-1062, 364-371. [CrossRef]

11. Reyes-Jurado, F.; Franco-Vega, A.; Ramírez-Corona, N.; Palou, E.; López-Malo, A. Essential Oils: Antimicrobial Activities, Extraction Methods, and Their Modeling. Food Eng. Rev. 2014, 7, 1-23. [CrossRef]

12. Catchpole, O.J.; Tallon, S.J.; Eltringham, W.E.; Grey, J.B.; Fenton, K.A.; Vagi, E.M.; Vyssotski, M.V.; Mackenzie, A.N.; Ryan, J.; Zhu, Y. The extraction and fractionation of specialty lipids using near critical fluids. J. Supercrit. Fluids 2009, 47, 591-597. [CrossRef]

13. Chaintreau, A. Simultaneous distillation-extraction: From birth to maturity-Review. Flavour Fragr. J. 2001, 16, 136-148. [CrossRef]

14. Lee, S.J.; Ahn, B. Comparison of volatile components in fermented soybean pastes using simultaneous distillation and extraction (SDE) with sensory characterisation. Food Chem. 2009, 114, 600-609. [CrossRef]

15. Guan, W.; Li, S.; Yan, R.; Huang, Y. Comparison of composition and antifungal activity of Artemisia argyi Lévl. et Vant inflorescence essential oil extracted by hydrodistillation and supercritical carbon dioxide. Nat. Prod. Lett. 2015, 20, 992-998. [CrossRef] 
16. Ge, Y.-B.; Wang, Z.-G.; Xiong, Y.; Huang, X.-J.; Mei, Z.-N.; Hong, Z.-G. Anti-inflammatory and blood stasis activities of essential oil extracted from Artemisia argyi leaf in animals. J. Nat. Med. 2016, 70, 531-538. [CrossRef] [PubMed]

17. Mevy, J.P.; Bessiere, J.M.; Rabier, J.; Dherbomez, M.; Ruzzier, M.; Millogo, J.; Viano, J. Composition and antimicrobial activities of the essential oil of Triumfetta rhomboidea Jacq. Flavour Fragr. J. 2010, 21, 80-83. [CrossRef]

18. Deng, Q.; Jie, S.; Zheng, M.; Xu, J.; Wan, C.; Huang, Q.; Qi, Z.; Guo, P.; Huang, F.; Lan, W. Thermal Stability of Rapeseed Oil Fortified with Unsaturated Fatty Acid Sterol Esters. J. Am. Oil Chem. Soc. 2014, 91, 1793-1803. [CrossRef]

19. Danh, L.T.; Han, L.N.; Triet, N.D.A.; Zhao, J.; Mammucari, R.; Foster, N. Comparison of Chemical Composition, Antioxidant and Antimicrobial Activity of Lavender (Lavandula angustifolia L.) Essential Oils Extracted by Supercritical $\mathrm{CO}_{2}$, Hexane and Hydrodistillation. Food Bioprocess Technol. 2013, 6, 3481-3489. [CrossRef]

20. Mohamed, A.A.; Ali, S.I.; El-Baz, F.K.; Hegazy, A.K.; Kord, M.A. Chemical composition of essential oil and in vitro antioxidant and antimicrobial activities of crude extracts of Commiphora myrrha resin. Ind. Crops Prod. 2014, 57, 10-16. [CrossRef]

21. Diao, W.-R.; Zhang, L.-L.; Feng, S.-S.; Xu, J.-G. Chemical composition, antibacterial activity, and mechanism of action of the essential oil from Amomum kravanh. J. Food Prot. 2014, 77, 1740. [CrossRef] [PubMed]

22. Sourmaghi, M.H.S.; Kiaee, G.; Golfakhrabadi, F.; Jamalifar, H.; Khanavi, M. Comparison of essential oil composition and antimicrobial activity of Coriandrum sativum L. extracted by hydrodistillation and microwave-assisted hydrodistillation. J. Food Sci. Technol. 2015, 52, 2452-2457. [CrossRef] [PubMed]

23. Liu, J.; Chen, P.; He, J.; Deng, L.; Wang, L.; Lei, J.; Rong, L. Extraction of oil from Jatropha curcas seeds by subcritical fluid extraction. Ind. Crops Prod. 2014, 62, 235-241. [CrossRef]

24. Zhu, Y.; Lv, H.P.; Dai, W.D.; Guo, L.; Tan, J.F.; Zhang, Y.; Yu, F.L.; Shao, C.Y.; Peng, Q.H.; Lin, Z. Separation of aroma components in Xihu Longjing tea using simultaneous distillation extraction with comprehensive two-dimensional gas chromatography-time-of-flight mass spectrometry. Sep. Purif. Technol. 2016, 164, 146-154. [CrossRef]

25. Lou, Z.; Chen, J.; Yu, F.; Wang, H.; Kou, X.; Ma, C.; Zhu, S. The antioxidant, antibacterial, antibiofilm activity of essential oil from Citrus medica L. var. sarcodactylis and its nanoemulsion. LWT Food Sci. Technol. 2017, 80, 371-377. [CrossRef]

26. Deng, J.; He, B.; He, D.; Chen, Z. A potential biopreservative: Chemical composition, antibacterial and hemolytic activities of leaves essential oil from Alpinia guinanensis. Ind. Crops Prod. 2016, 94, 281-287. [CrossRef]

Sample Availability: The Leaves of $A$. argyi Lévl. et Vant were purchased from A. argyi farm (Qichun, China). Artemisia argyi Lévl. et Vant essential oils are available from the authors. 